\title{
Проектирование программной платформы полного издательского цикла для издания сетевого мультимедийного журнала
}

\author{
Н.В. Борисов ${ }^{1,2}$, В.В. Захаркина ${ }^{1}$, И.А. Мбого ${ }^{1,2}$, Д.Е. Прокудин ${ }^{1,2}$ \\ ${ }^{1}$ Санкт-Петербургский государственный университет, Россия \\ ${ }^{2}$ Университет ИТМО, Россия \\ nikborisov@gmail.com, zakharkina@gmail.com, \\ irina.mbogo@gmail.com, hogben.young@gmail.com
}

\begin{abstract}
Аннотация. В настоящей статье описывается подход к проектированию программной платформы полного издательского цикла, в которой будет реализована функциональность, позволяющая как осуществлять все процессы, связанные с редакционно-издательской деятельностью через адаптивные веб-интерфейсы без привлечения дополнительных средств и выхода за пределы информационного пространства платформы; так и производить вёрстку, публикацию и отображение муьтимедийного контента через инструменты, являющиеся неотъемлемой частью платформы и реализованные через соответствующие интерфейсы. Кроме этого важной частью проектируемой системы должна стать реализация обмен метаданными, представленными в формате Dublin Core, с внешними информационными системами по протоколу ОАІ-РМН. Что позволит решать задачу оперативного распространения информации через современные информационные средства.
\end{abstract}

Ключевые слова: проектирование, мультимедийная информационная система, электронный сетевой мультимедийный журнал, информационная модель, информационные процессы, полный издательский цикл

\section{Введение}

Активно развивающаяся парадигма цифровой экономики, переход к шестому экономическому укладу вовлекает в орбиту информатизации различные виды деятельности человека в обществе, предлагая новые подходы к их организации и осуществления. Цифровые, информационно-коммуникационные технологии изменяют, в том числе, и такие традиционные виды деятельности как издательскую деятельность. При этом возникают такие формы изданий, как электронные книги, электронные сетевые журналы. Издание периодики в электронной форме не только упрощает и удешевляет редакционно-издательский процесс, но и позволяет привлекать в издания новые цифровые формы представления информации - графику, видео, мультимедиа и т.д. Это, в свою очередь, расширяет возможности оперативно распространять не только текстовые описания со статичными иллюстрациями, но и делать объектами статей различные мультимедийные объекты. Новые возможности представления различного цифрового контента делают такие журналы всё более востребованными и привлекательными у современного читателя, 
привыкшего воспринимать информацию с экрана. И, прежде всего, это относится к научным сетевым журналам, которые призваны распространять результаты исследований и разработок в области применения мультимедийного контента.

\section{Решения для издания сетевых журналов с поддержкой публикации мультимедийных материалов}

Мультимедийный контент широко используется для представления результатов научных исследований в различных областях науки. Он позволяет комплексно и наглядно представлять результаты численных расчетов, моделирования, реконструкций объектов и процессов.

Сложившаяся система научных публикаций, основанная на публикации текстов с иллюстрациями, не может полностью представить результаты исследований, полученные в мультимедийной форме. Эту задачу возможно решить проектированием и реализацией информационных систем электронных сетевых мультимедийных журналов. Однако, для их широкого внедрения в практику научных публикаций они должны поддерживать издательский процесс на всех его этапах, а также давать средства удобной работы с мультимедийным контентом на всех этапах издательского процесса и предоставлять читателям удобные средства его просмотра.

В настоящее время разработаны и находят практическое применение программные платформы, предназначенные для издания электронных сетевых журналов или электронных версий печатных периодических изданий. Самым известным и популярным решением является платформа полного издательского цикла для издания электронных сетевых журналов Open Journal Systems (OJS) [1, 3, 6, 9], которая является свободно распространяемым программным обеспечением с открытым кодом. Помимо реализации полного издательского цикла к преимуществам OJS можно отнести:

1) возможность автоматически включать публикуемые материалы в электронные индексируемые научные базы данных через реализацию протокола обмена метаданными OAI-PMH;

2) возможность экспортировать и выгружать статьи и метаданные в различных форматах для размещения их в различных сетевых сервисах (например, DOAJ, Erudit, CrossRef и др.);

3) предоставлять пользователям журнала возможность отслеживать публикуемые новости (посредством подписки на ленты в форматах RSS/Atom);

4) интегрировать систему OJS c Google Analytics, являющейся системой для анализа траффика компании Google и предоставляющей мощный инструментарий анализа различных статистических данных, относящихся к изданию.

Однако, существенным недостатком этой платформы является то, что OJS не обладает инструментами публикации мультимедийного контента. Статьи могут быть опубликованы как в формате PDF, так и в формате HTML. Было установлено, что в статье, представленной в формате HTML, возможно включить как локально размещаемые на сервере журнала файлы, так и включать код со сторонних ресурсов (например, Youtube). Однако, в этой системе не предусмотрена реализация процессов вёрстки, публикации и отображения мультимедийного контента. К тому же процесс размещения статьи в том виде, как её задумал автор, не предусмотрен. Отсутствие соответствующих веб-интерфейсов переводит процессы вёрстки и публикации в ручной режим, который подразумевает наличие соответствующих знаний и умений у специалистов, занятых в редакционно-издательском процессе.

С другой стороны, существуют сетевые электронные журналы, публикующие мультимедийный контент, но их платформы не предполагают организацию полного издательского цикла, что было выявлено на основе анализа процесса подачи материалов авторами и технологий публикации и отображения мультимедийного контента. Наиболее удачным, как видится, является журнал «Научная визуализация» (http://sv-journal.org.). Однако, даже на страницах этого журнала для просмотра некоторых мультимедийных объектов (например, WRL, http://svjournal.org./example/index.html) требуется использование дополнительного программного обеспечения, установленного на компьютер читателя. Если же устройство, на котором происходит

International Culture \& Technology Studies, Vol. 2, No. 1 
чтение статьи не поддерживает это программное обеспечение, то просмотр соответствующего контента становится невозможным.

В других журналах (например, «Медиамузыка», http://mediamusic-journal.com) мультимедийный контент может быть размещён на сторонних ресурсах (например, https://www.youtube.com), представляющих собой сервисы публикации контента определённого вида (аудио, видео), а размещение на страницах журнала происходит посредством встраиваемого кода со ссылкой на внешний сервис. Такой подход чреват зависимостью от третьей стороны и идёт вразрез с необходимостью гарантированного долговременного хранения мультимедийного контента (контент по каким-либо причинам может быть удалён из сервиса или сам сервис перестанет существовать и т.д. и т.п.). Несомненный интерес представляют исследования, проводимые в Институте прикладной математики им. М.В. Келдыша Российской академии наук, по публикации анимации и видео в научной публикации [7]. Например, в «Препринтах ИПМ им. М.В. Келдыша» некоторые статьи сопровождаются видео аннотациями на страницах метаданных публикации (например, http://library.keldysh.ru/preprint.asp?id=2013-51). В рамках этих исследований решены задачи встраивания в статью в формате PDF видеоролика.

При этом подача статей в различные рассмотренные мультимедийные электронные сетевые журналы происходит самым простым способом - посредством передачи как рукописи статьи, так и сопутствующих мультимедийных материалов в сообщении электронной почты на адрес редакции. А затем уже сотрудники редакции будут размещать материалы на сайте журнала и компоновать из них мультимедийную статью, отстраняя от этого процесса автора. Также надо понимать, что существуют ограничения на размеры передаваемых по электронной почте файлов. Конечно, снять эти ограничения возможно используя сетевые сервисы файлообмена, но это только усложняет процессы коммуникации авторов и редакции журнала.

\section{Особенности организации редакционно-издательского цикла при публикации мультимедийных статей}

В нашем понимании мультимедийная статья не является результатом механической конвертации печатного варианта в веб-формат (HTML). Даже в, казалось бы, наиболее простом случае, когда статья содержит лишь текст и иллюстрирующие изображения, можно достичь принципиально нового качества восприятия за счёт организации изобразительного материала на базе программных модулей, обеспечивающих требуемую интерактивность. Читатель может «пролистывать» миниатюры, выбирать изображения для вывода в полноэкранном режиме, просматривать фрагменты изображений высокого разрешения в увеличенном масштабе и т.д. Автор же получает возможность публикации объёмных коллекций изображений, количество и разрешение которых не ограничено жёсткими рамками печатного формата.

Мультимедийная статья в онлайн-издании включает не только такие характерные для печатного варианта элементы, как текст, таблицы, формулы, изображения, но и интерактивные мультимедийные элементы, реализация которых возможна лишь в электронном формате. В качестве примера можно привести:

- Галереи изображения с возможностью пролистывания, масштабирования и т.д.;

- аудио и видео объекты;

- интерактивная анимированная инфографика;

- 3D объекты и сцены;

- детализация просмотра изображений высокого разрешения и т.д.

Реализация всех аспектов организации медийного контента в рамках платформы мультимедийных онлайн-изданий предполагает целый ряд программных решений для различных ролей. Это не только представление интерактивных мультимедийных блоков в опубликованной статье, но и, например, соответствующие инструменты для авторов и технических редакторов («верстальщиков»). 
Концептуальной основой реализации мультимедийного сетевого электронного журнала является выделение медийных элементов как отдельных структурных сущностей статьи на уровне полей соответствующих таблиц базы данных. Такой подход не только обеспечивает гибкие варианты представления в опубликованной статье и эффективную работу технических редакторов. Весьма существенны и возможности для дальнейшей обработки контента статей агрегаторами.

Агрегаторы могут рассматривать не только статью в целом, но и её отдельные медийные элементы, которые (опционно) имеют свою дополнительную метаинформацию. Эти элементы могут быть, например, отобраны по запросу для представления во внешних коллекциях с сохранением привязки к статье. Такой подход выделения медийных элементов на уровне базовой структуры может лечь в основу инструментов, обеспечивающих фиксацию приоритета их публикации.

\section{Разработка интерфейса для редактирования мультимедийной статьи}

Существенным аспектом издательской онлайн-платформы является взаимодействие авторов с редакционной коллегией. Все известные коллективу авторов издательские онлайнплатформы реализуют этот аспект издательского цикла следующим образом.

Авторы проходят онлайн-регистрацию, заполняют свой профиль и вносят метаданные. Именно эта информация на всех стадиях доступна для просмотра и редактирования соответствующими ролями через веб-интерфейс. Взаимодействие авторов и редакционной коллегии в процессе работы над статьей происходит на уровне обмена файлами, которые в дальнейшем обрабатываются локально с использованием соответствующих прикладных программ, установленных на компьютере технического редактора. В этом случае публикация сложного мультимедийного контента может быть реализована при наличии высококвалифицированного разработчика-программиста в штате издательства. Альтернативой является разработка инструментария, позволяющего техническому персоналу без специального образования осуществлять верстку материала через веб-интерфейсы. В предлагаемом подходе предполагается разработать инструментарий, позволяющий осуществлять работу над статьей через веб-интерфейс. Так, соответствующие роли на разных стадиях могут просматривать и редактировать как текст, так и мультимедийные элементы статьи. Дополнительным позитивным следствием верстки через веб-интерфейсы является возможность просмотра и редактирования статьи соответствующими ролями на всех стадиях издательского цикла. А динамическое формирование программного кода, обеспечивающего функциональность мультимедийных блоков, предполагается включить в ядро планируемой к разработке системы.

В проектируемом решении предлагается реализовать следующий алгоритм подачи рукописи статьи в редакцию журнала (рис. 1). Подача осуществляется автором через личный кабинет после авторизации. Все действия сопровождаются направлением различных уведомлений на электронную почту участников редакционно-издательского цикла в соответствии с их ролями, реализованными в системе. После получения уведомления об отправки рукописи редактором или менеджером журнала принимается первичное решение о принятии рукописи к рассмотрению. В случае принятии статья рукописи к рассмотрению определяется её тип. Если статья не мультимедийная, то редактор назначает редактора раздела, который далее будет сопровождать прохождение рукописи с использованием стандартного редакционно-издательского процесса. При определении типа статьи как мультимедийной автору с уведомлением направляется предложение самому оформить статью для дальнейшего рецензирования (со ссылкой на страницу с рукописью, где необходимо отметить своё решение). Если автор выбирает самостоятельное оформление статьи (через личный кабинет), то происходит блокировка внесения любых изменений в он-лайн оформление статьи (включая набор файлов) любыми другими пользователями журнала (редактор, редактор раздела и т.д.) кроме автора. О решении автора направляется уведомление редактору. При этом автору становится доступен визуальный редактор, с помощью которого он оформляет статью. После окончания редактирования автор утверждает решение об окончании редактирования и передаёт статью на рецензирование (переход к стандартному процессу). В случае отказа автора от самостоятельного редактирования статьи направляется уведомление об этом редактору и менеджеру. При этом происходит блокировка внесения любых изменений в онлайн оформление статьи (включая набор файлов) любыми другими пользователями журнала (редактор, редактор раздела и т.д.) кроме менеджера. А менеджеру становится доступен

International Culture \& Technology Studies, Vol. 2, No. 1 
визуальный редактор, с помощью которого он оформляет статью. После завершения редактирования мультимедийной статьи менеджер делает отметку об этом, о чём направляется уведомление автору. Если он согласен с окончательным вариантом, то утверждает решение об окончании редактирования и передаёт статью на рецензирование (переход к стандартному процессу), иначе отправляет с комментарием на доработку менеджеру. Менеджер после получения уведомления дорабатывает статью и после утверждения решения о передачи статьи на рецензирование фиксируется дата поступления статьи в редакцию.

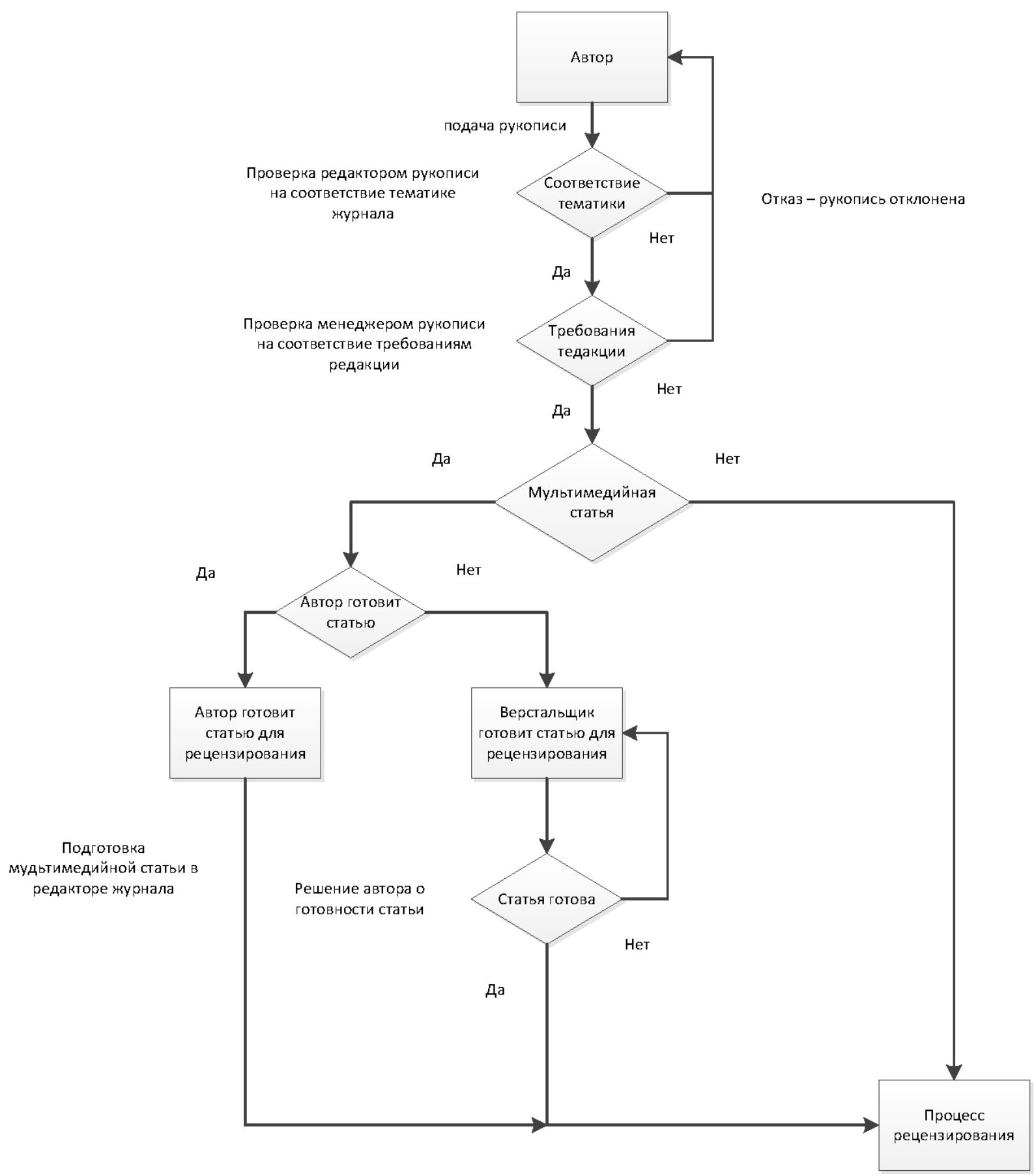

Рис 1. Алгоритм подачи статьи через сервис сайта журнала 


\section{Реализация прототипа программной платформы для издания сетевого мультимедийного журнала}

Новизна предлагаемого к реализации проекта состоит в объединении в одной программной платформе как функции обеспечения полного издательского цикла электронного сетевого журнала через, так и обеспечения возможности публикации в материалах журнала разнообразного мультимедийного контента посредством инструментария, позволяющего осуществлять работу над статьей через веб-интерфейс на всех этапах издательского цикла.

В рамках предлагаемого проекта предполагается решить следующие основные задачи:

1) разработать модели полного издательского цикла сетевого электронного журнала и его программная реализация;

2) реализовать процессы публикации и отображения мультимедийного контента в материалах электронного сетевого журнала;

3) создать инструменты отображения мультимедиа контента. В качестве мультимедиа предполагается публикация следующих материалов: коллекции изображений, аудио, видео, видео360, 3d модели и др.;

4) разработка инструментария, позволяющего техническому персоналу без специального образования осуществлять верстку материала через веб-интерфейсы;

5) реализация механизма долговременного гарантированного хранения мультимедийного контента;

6) реализация механизма обмена метаданными с внешними информационными системами;

7) реализация инструментария формирования информации для предоставления в библиографические информационные системы.

Проект предполагается реализовать на основе программного обеспечения (ПО) с открытым кодом и свободного ПО, системы управления контентом Drupal. В процессе создания мультимедийного электронного журнала будут использоваться как готовые инструменты и модули Drupal, ускоряющие процесс разработки, так и создаваться новые программные модули на основании API Drupal и независимые программы.

В разработанной программной платформе планируется реализовать функциональность, которая позволит:

- осуществлять все процессы, связанные с редакционно-издательской деятельностью через адаптивные веб-интерфейсы без привлечения дополнительных средств и выхода за пределы информационного пространства платформы. При этом основными сущностями системы являются выпуски и статьи. Статьи имеют внутренние состояния (в редакции, на рецензии, опубликовано и т.д.) и возможность перехода между состояниями. Возможности чтения и редактирования статей, написание рецензии, перевод из одного состояния в другое, публикация выпуска осуществляется на основе разграничения прав пользователей и ролей;

- осуществлять вёрстку, публикацию и отображения мультимедийного контента через инструменты, являющиеся неотъемлемой частью платформы и реализованные через соответствующие;

- обеспечить долговременное гарантированное хранение мультимедийного контента посредством разделения программной платформы на сервер приложений и файловый сервер для хранения мультимедийного контента с соответствующим инструментарием доступа к нему;

- реализовать обмен метаданными, представленными в формате Dublin Core, с внешними информационными системами по протоколу ОАІ-PMH.

Предполагается, что все материалы должны храниться у издателя. Такой подход требует разработки целого комплекса программных, технологических и инфраструктурных решений:

International Culture \& Technology Studies, Vol. 2, No. 1 
- разработка удобных механизмов добавления контента через web-интерфейс;

- разработка механизмов автоматизации вставки мультимедиа материалов в текст статьи в требуемое место;

- создание хранилища материалов;

- создание инструментов показа мультимедиа контента (в качестве мультимедиа предполагается публикация следующих материалов: коллекции изображений, аудио, видео, видео360, 3d модели и др.).

\section{Проектирование дополнительных возможностей и дальнейшая разработка}

Развитие современного информационного пространства в совокупности с постоянно увеличивающимися объёмами цифровой информации ставят актуальную проблему оперативного распространения актуальной информации, к которой, в том числе, относятся результаты научных исследований и технологических разработок. В связи с этим дальнейшее развитие информационной системы электронного сетевого мультимедийного журнала видится в проектировании и реализации соответствующих инструментов. В связи с чем предполагается, что каждая статья журнала будет иметь метаданные в соответствии с международной нотацией Dublin Core $[2,4,5,8]$. Это позволит обеспечить взаимодействие и интеграцию на уровне метаданных с другими коллекциями, поисковыми системами и сборщиками данных (агрегаторами). Материалы коллекции будут иметь механизм формирования метаданных о записях в соответствии с протоколом OAI-PMH, OpenURL. А реализация модуля провайдера OAI-PMH позволит автоматизировать процесс обмена метаданными с внешними информационными системами.

Также в планах интегрировать в программную платформу механизмов как полнотекстового поиска по журналу с использованием инструментов текстового анализа и языком запросов (одним из инструментов: ApacheSolr, Yandex или Google), так и параметрического поиска по метаданным с последующей многоуровневой и многофакторной фильтрацией результатов, что позволит более эффективно использовать журнал в поисковой деятельности.

Разработанную в дальнейшем платформу предполагается распространять на условиях программного обеспечения с открытым программным кодом совершенно безвозмездно. Это позволит в дальнейшем независимым разработчикам и программистам как улучшать основной функционал и инструментарий платформы, так и реализовывать новые функции в соответствии с потребностями конкретных решений.

\section{Литература}

[1] Bakht S., Hovey P., McLeran A. Open Journal Systems // Transliteracies Project. 2009. URL: http://transliteracies.english.ucsb.edu/post/research-project/research-clearinghouse-individual/researchreports/open-journal-systems-2 (дата обращения: 12.03.2017).

[2] Dublin Core Metadata for Resource Discovery // The Internet Engineering Task Force. URL: http://www.ietf.org/rfc/rfc2413.txt (дата обращения: 12.03.2017).

[3] Edgar B.D., Willinsky J. A Survey of the Scholarly Journals Using Open Journal Systems // Scholarly and Research Communication. URL: http://pkp.sfu.ca/files/OJS\%20Journal\%20Survey.pdf (дата обращения: 12.03.2017).

[4] The Dublin Core metadata element set. The International Organization for Standardization. URL: https://www.iso.org/obp/ui/\#iso:std:iso:15836:ed-2:v1:en (дата обращения: 12.03.2017).

[5] The Dublin Core Metadata for Simple Resource Discovery // Dublin Core Metadata Initiative. URL:http://dublincore.org/documents/ (дата обращения: 12.03.2017).

[6] Willinsky J. Open Journal Systems: An example of Open Source Software for journal management and publishing // Library Hi-Tech. 2005. Vol. 23, № 4. P. 504-519. URL: http://pkp.sfu.ca/files/Library Hi Tech DRAFT.pdf (дата обращения: 12.03.2017).

[7] Горбунов-Посадов М.М., Ролдугин Д. С., Слепенков М.И., Тузов И.В. Анимация и видео в научной публикации // Препринты ИПМ им. М.В.Келдыша. 2014. No 104.32 с. URL: http://library.keldysh.ru/preprint.asp?id=2014-104 (дата обращения: 12.03.2017).

[8] Общие форматы метаданных. Dublin Core // Российские Электронные библиотеки. URL: http://www.elbib.ru/index.phtml?env_page=methodology/metadata/md_review/md_descrip_general.html (дата обращения: 12.03.2017). 
[9] Прокудин Д.Е. Через открытую программную издательскую платформу к интеграции в мировое научное сообщество: решение проблемы оперативной публикации результатов научных исследований // Научная периодика: проблемы и решения. 2013. № 6 (18). C. 13-18. DOI: http://dx.doi.org/10.18334/np36109.

\title{
Design of software platform full publishing cycle for the publication of online multimedia journal
}

\author{
N.V. Borisov ${ }^{1,2}$, V.V. Zakharkina ${ }^{1}$, I.A. Mbogo ${ }^{1,2}$, D.E. Prokudin ${ }^{1,2}$ \\ ${ }^{1}$ Saint-Petersburg State University, Russia \\ ${ }^{2}$ ITMO University, Russia
}

\begin{abstract}
This article describes an approach to the design of software platform full publishing cycle, which will be implemented a functionality that allow to carry out all the processes associated with the publishing activities through responsive web interfaces without attraction of additional means and output outside the information space of the platform; and producing the layout, publication and display multimedia content through tools that are an integral part of the platform and implemented via appropriate interfaces. In addition, an important part of the designed system must be to implement the exchange of metadata represented in the Dublin Core format, with external information systems according to the Protocol OAI-PMH. That will allow solving the problem of prompt dissemination of information through modern information tools.
\end{abstract}

Keywords: design, multimedia information system, online multimedia journal, information model, information processes, full publishing cycle.

\section{References}

[1] Bakht S., Hovey P., McLeran A. (2009). Open Journal Systems. Transliteracies Project. Available at: http://transliteracies.english.ucsb.edu/post/research-project/research-clearinghouse-individual/researchreports/open-journal-systems-2 (access date: 12/3/2017).

[2] Dublin Core Metadata for Resource Discovery. The Internet Engineering Task Force. Available at: http://www.ietf.org/rfc/rfc2413.txt (access date: 12/3/2017).

[3] Edgar B.D., Willinsky J. A Survey of the Scholarly Journals Using Open Journal Systems. Scholarly and Research Communication. Available at: http://pkp.sfu.ca/files/OJS\%20Journal\%20Survey.pdf (access date: 12/3/2017).

[4] The Dublin Core metadata element set. The International Organization for Standardization. Available at: https://www.iso.org/obp/ui/\#iso:std:iso:15836:ed-2:v1:en (access date: 12/3/2017).

[5] The Dublin Core Metadata for Simple Resource Discovery. Dublin Core Metadata Initiative. http://dublincore.org/documents/ (access date: 12/3/2017).

[6] Willinsky J. (2005). Open Journal Systems: An example of Open Source Software for journal management and publishing. Library Hi-Tech. 23(4). 504-519. Available http://pkp.sfu.ca/files/Library_Hi_Tech_DRAFT.pdf (access date: 12/3/2017).

[7] Gorbunov-Possadov M. M., Roldugin D. S., Slepenkov M.I., Tuzov I.V. (2014). Animation and video in scientific publication. KIAM Preprint. № 104. Available at: http://library.keldysh.ru/preprint.asp?id=2014104 (access date: 12/3/2017).

[8] Common metadata formats. Dublin Core. Russian Digital Libraries. Available at: http://www.elbib.ru/index.phtml?env_page=methodology/metadata/md_review/md_descrip_general.html (access date: 12/3/2017).

[9] Dmitriy Prokudin (2013). Through an open software publishing platform for integration into the global scientific community: addressing rapid publication of research results. Scholarly Communication Review, 3(6). 13-18. DOI: http://dx.doi.org/10.18334/np36109. 